\title{
Dynamics of a double-stranded DNA segment in a shear flow
}

\author{
Debabrata Panja \\ Institute for Theoretical Physics, Universiteit Utrecht, Leuvenlaan 4, 3584 CE Utrecht, The Netherlands \\ Gerard T. Barkema \\ Institute for Theoretical Physics, Universiteit Utrecht, Leuvenlaan 4, 3584 CE Utrecht, The Netherlands \\ and Instituut-Lorentz, Universiteit Leiden, Niels Bohrweg 2, 2333 CA Leiden, The Netherlands
}

J. M. J. van Leeuwen

Instituut-Lorentz, Universiteit Leiden, Niels Bohrweg 2, 2333 CA Leiden, The Netherlands

(Received 24 December 2015; published 7 April 2016)

\begin{abstract}
We study the dynamics of a double-stranded DNA (dsDNA) segment, as a semiflexible polymer, in a shear flow, the strength of which is customarily expressed in terms of the dimensionless Weissenberg number Wi. Polymer chains in shear flows are well known to undergo tumbling motion. When the chain lengths are much smaller than the persistence length, one expects a (semiflexible) chain to tumble as a rigid rod. At low Wi, a polymer segment shorter than the persistence length does indeed tumble as a rigid rod. However, for higher Wi the chain does not tumble as a rigid rod, even if the polymer segment is shorter than the persistence length. In particular, from time to time the polymer segment may assume a buckled form, a phenomenon commonly known as Euler buckling. Using a bead-spring Hamiltonian model for extensible dsDNA fragments, we first analyze Euler buckling in terms of the oriented deterministic state (ODS), which is obtained as the steady-state solution of the dynamical equations by turning off the stochastic (thermal) forces at a fixed orientation of the chain. The ODS exhibits symmetry breaking at a critical Weissenberg number $\mathrm{Wi}_{\mathrm{c}}$, analogous to a pitchfork bifurcation in dynamical systems. We then follow up the analysis with simulations and demonstrate symmetry breaking in computer experiments, characterized by a unimodal to bimodal transformation of the probability distribution of the second Rouse mode with increasing Wi. Our simulations reveal that shear can cause strong deformation for a chain that is shorter than its persistence length, similar to recent experimental observations.
\end{abstract}

DOI: 10.1103/PhysRevE.93.042501

\section{INTRODUCTION}

The flow properties of a solution of polymers have attracted the interest of physicists for a long time. One side of the problem concerns how the concentration of dissolved polymers influences, e.g., the viscous (or viscoelastic) properties of the fluid. The other side concerns how fluid flow influences the behavior of polymers. Here we restrict ourselves to the latter. Specifically, we consider a dilute solution of double-stranded DNA (dsDNA) segments in water under shear. Double-stranded DNA is a semiflexible polymer, since it preserves mechanical rigidity over a range, characterized by the persistence length $l_{p} \approx 40 \mathrm{~nm}[1,2]$, along its contour.

That a polymer will go through a "coil-stretch transition" under the influence of a shear flow was originally predicted by de Gennes [3], although it would be more than two decades before the coil-stretch transition would be put to experimental verification. Interestingly, however, the first key experiment along this line-combining fluid flow and fluorescence microscopy techniques (the latter in order to visually track polymers) — was performed to determine the force-extension curve of dsDNA, wherein uniform water flow was used to stretch (end-tethered) polymers [4]. Extending that experimental setup to include more complicated flow patterns, such as elongational flow [5,6] and shear flow [7,8] soon followed, driven by the quest to understand how flow-induced conformational changes take place in polymers (see, e.g., Ref. [9] for a review).

An intriguing by-product of the experiments with shear flow was the tumbling motion of the chains, which can be tracked by, e.g., the relative orientation of the polymer's end-to-end vector with respect to the direction of the flow [7,8]. Although irregular at short time scales, a tumbling frequency could be defined based on the long-time statistics of the chain's orientation. The tumbling behavior soon started to receive further attention from researchers: Over the last decade and a half, a number of models have been constructed [10-12] and further experiments have been performed [13-16] to characterize and quantify the tumbling behavior, in particular, the dependence of the tumbling frequency on the shear strength. The subject of this paper, too, is tumbling behavior in a shear flow, specifically for a dsDNA chain that is smaller than its persistence length.

As stated earlier, the shear strength $\dot{\gamma}$ is customarily expressed by the dimensionless Weissenberg number $\mathrm{Wi}=$ $\dot{\gamma} \tau$, where $\tau$ is a characteristic time scale for the polymer. At one extreme, for flexible polymers (polymer segments that are many times longer than their persistence length, assuming coil configurations in the absence of shear), which many of the above studies focus on, the natural choice for $\tau$ is the polymer's terminal relaxation time. For them there is good theoretical, numerical, and experimental evidence that the tumbling frequency $f$ scales with $\mathrm{Wi}$ as $f \propto \mathrm{Wi}^{2 / 3}$ [10,11,13-15]. At the other extreme, for semiflexible polymer segments (polymer segments shorter than their persistence lengths resemble the configuration of rigid rods in the absence of shear, and the natural choice of $\tau$ is the time scale for rotational diffusion of a rigid rod of the same length), one expects the rigid-rod result, namely, that the tumbling 
frequency scales as $f \propto \mathrm{Wi}^{2 / 3}$ [16-18]. (Given that the physics of tumbling is different for flexible and semiflexible polymers, the similarity in the scaling behavior of $f$ is striking.)

Recently, Harasim et al. [16] experimented with tumbling f-actin segments of several lengths $(\sim 3-40 \mu \mathrm{m})$ in a shear flow. They found that the tumbling frequency $f$ follows the law $f \propto$ $\mathrm{Wi}^{2 / 3}$ for small Weissenberg numbers. A closer inspection of their data reveals significant deviations from the $f \propto \mathrm{Wi}^{2 / 3}$ power law around and above the persistence length $(\approx 16 \mu \mathrm{m})$. Images and movies out of the experiments have revealed that f-actin segments of lengths smaller than the persistence length can strikingly buckle into $J$ and $U$ shapes, broadly known as Euler buckling.

These issues of buckling and the tumbling frequency were taken up by Lang et al. [12] by an extensive modeling study, using the inextensible wormlike chain as Hamiltonian. They discussed the tumbling frequency for the whole range spanning the two extremes, i.e., from flexible to semiflexible polymer segments, and reported, in the intermediate regime, the dependence $f \propto \mathrm{Wi}^{3 / 4}$.

The present paper has been inspired by the experiment of Harasim et al. [16]. Our focus is to provide a quantitative characterization of the Euler buckling, and the corresponding shapes of a tumbling semiflexible polymer segment in a shear flow. To this end, we take advantage of a recently developed bead-spring model for semiflexible polymers $[19,20]$ and its highly efficient implementation on a computer [21]. We model dsDNA segments dynamics for lengths $\lesssim 20 \mathrm{~nm}$, and analyze their dynamics in terms of the Rouse modes [22]. The persistence length of dsDNA is $\approx 40 \mathrm{~nm}$, corresponding to $\approx 120$ beads with the average intrabead distance $\approx 0.33 \mathrm{~nm}$, the length of a dsDNA base pair. We show that the tumbling frequency adheres to the rigid-rod results at low $\mathrm{Wi}$ and that for high $\mathrm{Wi}$, semiflexible polymer segments tumble much faster. This difference quickly leads us to issues related to (Euler) buckling of the chain under the influence of shear. We first analyze Euler buckling in terms of the oriented deterministic state (ODS), which results from turning off the stochastic (thermal) forces in polymer dynamics at a fixed orientation of the chain. In this state the internal forces, tending to keep the chain straight, balance the shear forces. Below a critical Weissenberg number $\mathrm{Wi}_{\mathrm{c}}$, the ODS shows a slightly bent $S$ shape. Above $\mathrm{Wi}_{\mathrm{c}}$ a symmetry breaking takes place, analogous to pitchfork bifurcation, where the ODS strongly deviates from a rigid rod.

We follow up the ODS analysis with simulations and demonstrate symmetry breaking in computer experiments, and demonstrate that, similar to the experimental snapshots found for f-actin filaments in Ref. [16], shear can cause strong deformation, even for a chain that is shorter than its persistence length.

The structure of the paper is as follows. In Sec. II we introduce the model. In Sec. III we describe the polymer dynamics in terms of the Rouse modes. In Sec. IV we analyze the time evolution of the orientation of the polymer, from which we determine the tumbling frequency. In Sec. V we analyze Euler buckling, identify the critical Weissenberg number $\mathrm{Wi}_{\mathrm{c}}$ and solve for the shapes of the polymer in the ODS. We follow up the theory of Sec. V with simulations in
Sec. VI, and end the paper with a discussion in Sec. VII. See the Supplemental Material [23] for a movie of a tumbling dsDNA segment—-details on the movie are provided in Sec. VI.

\section{THE MODEL}

The Hamiltonian for our bead-spring model for semiflexible polymers, the details of which can be found in our earlier works [19-21], reads

$$
\mathcal{H}=\frac{\lambda}{2} \sum_{n=1}^{N}\left(\left|\mathbf{u}_{n}\right|-d\right)^{2}-\kappa \sum_{n=1}^{N-1} \mathbf{u}_{n} \cdot \mathbf{u}_{n+1},
$$

with stretching and bending parameters $\lambda$ and $\kappa$, respectively. Here $\mathbf{u}_{n}$ is the bond vector between the $(n-1)$ th and the $n$th beads

$$
\mathbf{u}_{n}=\mathbf{r}_{n}-\mathbf{r}_{n-1},
$$

and $\mathbf{r}_{n}$ is the position of the $n$th bead $(n=0,1, \ldots, N)$. The parameter $d$ provides a length scale by the use of which we reduce the Hamiltonian to

$$
\frac{\mathcal{H}}{k_{B} T}=\frac{1}{T^{*}}\left[\sum_{n=1}^{N}\left(\left|\mathbf{u}_{n}\right|-1\right)^{2}-2 v \sum_{n=1}^{N-1} \mathbf{u}_{n} \cdot \mathbf{u}_{n+1}\right],
$$

with dimensionless $v=\kappa / \lambda$ and $T^{*}=k_{B} T /\left(\lambda d^{2}\right)$ parametrizing the Hamiltonian. In this formulation the persistence length of the polymer is given by $l_{p}=\left(v / T^{*}\right) d /(1-2 v)$. The model is a discrete version of the polymer with $N$ discretization units (i.e., of length $N$ ). From the analysis of the ground state of the Hamiltonian (3) [19-21], each discretization unit can be shown to have a length $a=d /(1-2 v)$.

The parameters of the model $-T^{*}$ and $\nu$-are determined by matching to the force-extension curve. For dsDNA, our semiflexible polymer of choice in this paper, we use $a=$ $0.33 \mathrm{~nm}$, the length of a dsDNA base pair, which leads to $T^{*}=0.034$ and $v=0.353$, meaning that one persistence length corresponds to $N \approx 120$ [19-21].

\section{POLYMER DYNAMICS}

\section{A. Construction of the Rouse modes modes}

We analyze the dynamics of the polymer by its Rouse modes, since they turn out to be a convenient scheme for solving the equations of motion with a sizable time step, without introducing large errors [21].

The representation of the configurations of a polymer chain in terms of its fluctuation modes uses basis functions. The well-known Rouse modes employ the basis functions

$$
\phi_{n, p}=\left(\frac{2}{N+1}\right)^{1 / 2} \cos \left[\frac{(n+1 / 2) p \pi}{N+1}\right],
$$

such that conversion of positions $\mathbf{r}_{n}$ to Rouse modes $\mathbf{R}_{p}$ and vice versa are given by

$$
\mathbf{R}_{p}=\sum_{n} \mathbf{r}_{n} \phi_{n, p}, \quad \mathbf{r}_{n}=\sum_{p} \phi_{n, p} \mathbf{R}_{p} .
$$

The modes with $p=0$ correspond to the location of the center of mass, the dynamics of which can be rigorously separated from that of the other modes. We eliminate the 
center-of-mass motion by always measuring the bead positions with respect to the center of mass.

\section{B. The equations for the Rouse modes under shear}

We consider the situation where water flows in the $\hat{\mathbf{x}}$ direction, with a shear gradient $\dot{\gamma}$ in the $\hat{\mathbf{y}}$ direction. The Langevin equation for the motion of the bead position $\mathbf{r}_{n}$ then reads

$$
\frac{d \mathbf{r}_{n}}{d t}=-\frac{1}{\xi} \frac{\partial \mathcal{H}}{\partial \mathbf{r}_{n}}+\dot{\gamma}\left(y_{n}-Y_{\text {c.m. }}\right) \hat{\mathbf{x}}+\boldsymbol{k}_{n} .
$$

The Hamiltonian $\mathcal{H}$ is given in Eq. (1), and $\xi$ is the friction coefficient due to the viscous drag, acting on each bead. The first term on the right hand side of the equation represents the internal force, which tends to keep the chain straight. The second term is the shear force due to the flow, where $\dot{\gamma}$ is the shear rate, $Y_{\mathrm{c} . \mathrm{m}}$. is the $y$ coordinate of the center of mass of the chain, and $y_{n}$ is the $y$ coordinate of monomer $n$. As mentioned earlier, we measure the bead positions with respect to the location of the chain's center of mass, leading to the term $\propto\left(y_{n}-Y_{\text {c.m. }}\right)$. The last term in Eq. (6) gives the influence of the random thermal force $\boldsymbol{k}_{n}$, which has the correlation function

$$
\left\langle k_{n}^{\alpha}(t) k_{m}^{\beta}\left(t^{\prime}\right)\right\rangle=\left(2 k_{B} T / \xi\right) \delta^{\alpha, \beta} \delta_{n, m} \delta\left(t-t^{\prime}\right) .
$$

In order to work with dimensionless units we replace the time $t$ with

$$
\tau=\lambda t / \xi .
$$

The ratio $\xi / \lambda$ then becomes the microscopic time scale, such that $\tau$ is dimensionless. In the same spirit we combine the shear ratio $\dot{\gamma}$ with this time scale, leading to the dimensionless constant $g$ as the shear strength

$$
g=\dot{\gamma} \frac{\xi}{\lambda} .
$$

The shear strength is customarily expressed in terms of the Weissenberg number, which we define as

$$
\mathrm{Wi}=\frac{\dot{\gamma}}{2 D_{r}} \quad \text { with } \quad D_{r}=\frac{k_{B} T}{I \xi} .
$$

Here $D_{r}$ is the rotational diffusion constant with $I$ as the moment of inertia of the polymer segment in its ground state (of the Hamiltonian). The relation between the two dimensionless quantities Wi and $g$ is then given by

$$
\mathrm{Wi}=g \frac{I_{0}}{2 T^{*}},
$$

where $I_{0}=I / d^{2}$, the dimensionless moment of inertia of the polymer segment in the ground state.

Using the orthogonal transformation converting positions into modes the dynamic equations for the Rouse modes can be cast in the form [21]

$$
\frac{d \mathbf{R}_{p}}{d \tau}=-\zeta_{p} \mathbf{R}_{p}+\mathbf{F}_{p}+\mathbf{H}_{p}+\boldsymbol{K}_{p} .
$$

For the decay constant we use the expression

$$
\zeta_{p}=4 v\left[1-\cos \left(\frac{p \pi}{N+1}\right)\right]^{2} .
$$

This spectrum follows from a subtraction in the coupling force $\mathbf{H}_{p}$, which derives from the contour length term in the Hamiltonian [21]

$$
\mathbf{H}_{p}=\left(\frac{2}{N+1}\right)^{1 / 2} \sum_{n} \sin \left(\frac{p n \pi}{N+1}\right) \mathbf{u}_{n}\left(\frac{1}{u_{n}}-1+2 v\right) .
$$

The subtraction $1-2 v$ within the last parentheses changes the Rouse spectrum from longitudinal to the transverse form Eq. (13). Finally, $\mathbf{F}_{p}$ is the shear force given by

$$
\mathbf{F}_{p}=g \hat{\mathbf{x}}\left(\hat{\mathbf{y}} \cdot \mathbf{R}_{p}\right) .
$$

The fluctuating thermal force $K_{p}^{\alpha}$ is the orthogonal transform of the $\boldsymbol{k}_{n}$ in Eq. (7),

$$
\left\langle K_{p}^{\alpha}(\tau) K_{q}^{\beta}\left(\tau^{\prime}\right)\right\rangle=2 T^{*} \delta^{\alpha, \beta} \delta_{p, q} \delta\left(\tau-\tau^{\prime}\right)
$$

Although the Rouse modes turn out to be a convenient scheme for solving the equations of motion with a sizable time step without large errors, we do pay a computational penalty in the calculation of the coupling force, which requires a transformation (8) from the Rouse modes to the bond vectors and the transformation (14) back to the modes. The penalty can be kept to the minimum by the use the fast Fourier transform (FFT) to switch from modes to bead positions; it keeps the number of operations of the order $N \log N$.

\section{Body-fixed coordinate system to analyze tumbling dynamics}

One of the major quantities of interest in the tumbling process is the dynamics of the orientation of the polymer. The orientation can be defined in several ways. The most common one is the direction of the end-to-end vector. Since the ends of the chain fluctuate substantially over short-time scales, this is not a slow variable. We prefer to use as orientation the direction of the first Rouse mode $\mathbf{R}_{1}$, which is the slowest decaying mode. We therefore define the orientation $\hat{\mathbf{n}}$ of the polymer as

$$
\hat{\mathbf{n}}=\hat{\mathbf{R}}_{1} \text {. }
$$

We refer to the components of the Rouse modes in the direction of $\hat{\mathbf{n}}$ as longitudinal components

$$
R_{p}^{l}=\hat{\mathbf{n}} \cdot \mathbf{R}_{p} .
$$

The perpendicular directions are transverse to $\hat{\mathbf{n}}$. The first Rouse mode has, by definition, only a longitudinal component. In practice, the so-defined orientation does not differ much from the direction of the end-to-end vector.

Further, it is convenient to discuss the temporal behavior of the polymer not only in the laboratory-frame coordinate system, with coordinate axes $(\hat{\mathbf{x}}, \hat{\mathbf{y}}, \hat{\mathbf{z}})$, but also in the body-fixed coordinate system which we define as follows. Along with the unit vector $\hat{\mathbf{n}}$, one of the two transverse axes, $\hat{\mathbf{n}}$, is taken perpendicular to $\hat{\mathbf{n}}$ and $\hat{\mathbf{x}}$, namely,

$$
\hat{\mathbf{m}}=\hat{\mathbf{n}} \times \hat{\mathbf{x}} / r, \quad r=\left[n_{y}^{2}+n_{z}^{2}\right]^{1 / 2} .
$$

The mode component in this direction, being perpendicular to $\hat{\mathbf{x}}$, is not influenced by the shear force. The other transverse direction is then naturally obtained as

$$
\hat{\mathbf{s}}=\hat{\mathbf{m}} \times \hat{\mathbf{n}} .
$$


Vector components along $\hat{\mathbf{s}}$ are maximally sheared. The system $(\hat{\mathbf{n}}, \hat{\mathbf{s}}, \hat{\mathbf{m}})$ forms an orthogonal basis set. For later use, below we list the Cartesian components of the vectors $(\hat{\mathbf{n}}, \hat{\mathbf{s}}, \hat{\mathbf{m}})$ :

$$
\begin{aligned}
& n_{x}=\sin \theta \cos \phi, \quad s_{x}=r, \quad m_{x}=0, \\
& n_{y}=\sin \theta \sin \phi, \quad s_{y}=-n_{x} n_{y} / r, \quad m_{y}=n_{z} / r, \\
& n_{z}=\cos \theta, \quad s_{z}=-n_{x} n_{z} / r, \quad m_{z}=-n_{y} / r .
\end{aligned}
$$

We denote the components of the modes generically with the index $\alpha$, which alternatively runs through $\alpha=(x, y, z)$ or $\alpha=$ $(n, s, m)$.

\section{TIME EVOLUTION OF THE ORIENTATION OF THE POLYMER}

The evolution of the orientation is given by the dynamics of the two transverse components of $\mathbf{R}_{1}$,

$$
\frac{d \hat{\mathbf{n}}}{d \tau}=\frac{d}{d \tau} \frac{\mathbf{R}_{1}}{R_{1}^{l}}=\frac{d \mathbf{R}_{1}}{d \tau} \frac{1}{R_{1}^{l}}+\mathbf{R}_{1} \frac{d}{d \tau} \frac{1}{R_{1}^{1}}=\frac{1}{R_{1}^{l}} \frac{d \mathbf{R}_{1}}{d \tau},
$$

wherein the third equality follows from the fact that the transverse components of $\mathbf{R}_{1}$ vanish by definition. So the temporal derivative of the longitudinal component is multiplied the vanishing transverse component. We then use Eq. (12) and get

$$
\frac{d \hat{\mathbf{n}}}{d \tau}=g \hat{\mathbf{x}}(\hat{\mathbf{y}} \cdot \hat{\mathbf{n}})+\left(\mathbf{H}_{1}+\boldsymbol{K}_{1}\right) / R_{1}^{l} .
$$

Obviously, in the right hand side of the equations only the transverse components of the vectors are relevant.

For the interpretation of Eq. (23) we note that $R_{1}^{l}$ is closely related to the moment of inertia $I$ of the chain, which is defined as

$$
\frac{I}{d^{2}}=\sum_{n}\left(r_{n}^{l}\right)^{2}=\sum_{p}\left(R_{p}^{l}\right)^{2}=\sum_{p} I_{p},
$$

where $I_{p}$ is the contribution of the $p$ th Rouse mode to the moment of inertia. When the chain is (relatively) straight, the sum over the modes is heavily dominated by the first component $I_{1}$. So it is an indicative approximation to replace $I$ by $I_{1}$.

In the (relatively) straight state the configuration of the chain resembles that of a straight rod. In order to make a connection with the equation of tumbling for a rigid rod, we rewrite the equation using a different scaling of the time $\tau$. We define the variable $\tilde{\tau}$, linked to the Weissenberg number, as

$$
\tilde{\tau}=g \tau / \mathrm{Wi}, \quad \text { with } g / \mathrm{Wi}=2 T^{*} / I_{1},
$$

where we have used $I_{1}$ as measure for the moment of inertia. In terms of $\tilde{\tau}$, Eq. (23) then becomes

$$
\frac{d \hat{\mathbf{n}}}{d \tilde{\tau}}=\operatorname{Wi}[\hat{\mathbf{x}}(\hat{\mathbf{y}} \cdot \hat{\mathbf{n}})]+\left(\tilde{\mathbf{H}}_{1}+\tilde{\boldsymbol{K}}_{1}\right)
$$

with $\tilde{\mathbf{H}}_{1}$ and $\tilde{\mathbf{G}}_{1}$ defined as

$$
\tilde{\mathbf{H}}_{1}=\frac{\sqrt{I_{1}}}{2 T^{*}} \mathbf{H}_{1}, \quad \tilde{\boldsymbol{K}}_{1}(\tilde{\tau})=\frac{\sqrt{I_{1}}}{2 T^{*}} \boldsymbol{K}_{1}(\tau) .
$$

The new random force has a correlation function

$$
\left\langle\tilde{K}_{1}^{\alpha}(\tilde{\tau}) \tilde{K}_{1}^{\beta}\left(\tilde{\tau}^{\prime}\right)\right\rangle=\delta^{\alpha, \beta} \delta\left(\tilde{\tau}-\tilde{\tau}^{\prime}\right) .
$$

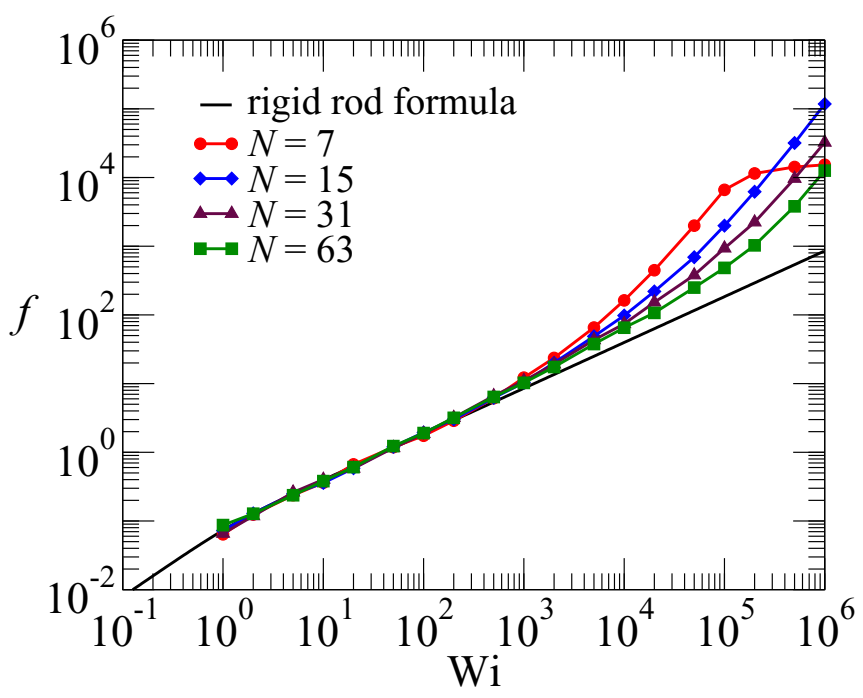

FIG. 1. The tumbling frequency as a function of the Weissenberg number Wi for a series of chain lengths $N=7,15,31$, and 63 . The conversion to Weissenberg numbers is based on the ground-state moment of inertia [see Eq. (11)].

Apart from the mode-coupling term $\tilde{\mathbf{H}}_{1}$, Eq. (26) is the same as that of an infinitely thin rigid rod [18]. Therefore, it makes sense to compare the tumbling frequency $f$ with that of the rigid rod, given by [18]

$$
f=\frac{\mathrm{Wi}}{4 \pi\left(1+0.65 \mathrm{Wi}^{2}\right)^{1 / 6}} .
$$

For comparison we show in Fig. 1 the tumbling frequency of dsDNA chains for several lengths shorter than the persistence length (which corresponds to $N \approx 120$ ), as found from simulations, together with that of a rigid-rod expression, Eq. (29). The simulations have been performed using an efficient implementation of semiflexible polymer dynamics [21] of the bead-spring model $[19,20]$. At any given value of the Weissenberg number, obtained by using the rotational inertia of a rigid rod that has the same configuration as the ground state of the Hamiltonian (3), snapshots of the polymer have been used to calculate its orientation $[\theta(t), \phi(t)]$ in the laboratory frame. The $\phi(t)$ data are then fitted by a straight line to obtain the tumbling frequency.

We point out that the simulations follow the rigid-rod formula for a surprisingly large range of Weissenberg numbers, clearly indicating that up to $\mathrm{Wi}=100$ the mode-coupling force $\tilde{\mathbf{H}}_{1}$ is unimportant. In order to see what this implies for the shear rate $\dot{\gamma}$, using the expression $I / d^{2}=N^{3} / 12$ for the moment of inertia, we write the relation between $\mathrm{Wi}$ and the shear rate $\dot{\gamma}$ as

$$
\mathrm{Wi}=\dot{\gamma} \frac{a^{2} \xi}{k_{B} T} \frac{N^{3}}{24} .
$$

Note that in Eq. (30) the molecular time scale equals [21]

$$
\frac{a^{2} \xi}{k_{B} T}=52 \times 10^{-12} \mathrm{~s} .
$$

Commercially available rheometers at present are limited to shear rates $\dot{\gamma}<10^{6} \mathrm{~s}^{-1}$. This implies, for dsDNA fragments 
of the order of the persistence length, say, $N=100$, that only the range $\mathrm{Wi}<2$ is presently achievable in the laboratory; i.e., the differences from the rigid-rod behavior in Fig. 1 lie outside the reach of present day experiments. Nevertheless, the origin of the deviations from the rigid-rod behavior is theoretically interesting; we will address this issue in the Sec. VI.

\section{SHAPES OF SEMIFLEXIBLE POLYMER IN THE ORIENTED DETERMINISTIC STATE}

In order to further analyze the tumbling process, it is useful to note that the orientation changes at a slower rate than all the other modes. This prompts us to focus on the configuration which is obtained as the steady-state solution of the dynamical equations by turning off the stochastic (thermal) forces at a fixed orientation of the chain. We call this configuration the oriented deterministic state (ODS). We use the properties of the ODS as indicative for the configurations of the chain at the given orientation.

\section{A. The approach to the oriented deterministic state (ODS)}

The ODS configuration of the chain is obtained from Eq. (12) by the decay of the equation

$$
\frac{d \mathbf{R}_{p}}{d \tau}=-\zeta_{p} \mathbf{R}_{p}+\mathbf{F}_{p}+\mathbf{H}_{p} .
$$

The constraint of a fixed orientation is imposed by leaving out the transverse components of the mode $\mathbf{R}_{1}$ and setting them equal to zero in the other mode equations. Asymptotically the configuration obeying Eq. (32) will turn into the ODS. So for the ODS the left hand side of Eq. (32) vanishes. The approach to the ODS configuration as following from Eq. (32) is slow.

A further simplification of finding the asymptotic state of Eq. (32) follows by considering the ODS in the body-fixed system. As there are no shear forces in the $\hat{\mathbf{m}}$ direction, the ODS shape has no component in that direction. For the two other equations in the $(\hat{\mathbf{n}}, \hat{\mathbf{s}})$ plane we get in detail

$$
\begin{gathered}
\left(\zeta_{p}-g n_{x} n_{y}\right) R_{p}^{n}-g n_{x} s_{y} R_{p}^{s}=H_{p}^{n}, \\
g s_{x} n_{y} R_{p}^{n}+\left(\zeta_{p}-g s_{x} s_{y}\right) R_{p}^{s}=H_{p}^{s} .
\end{gathered}
$$

For $p=1$ we have only the first equation since the second refers to the transverse component $R_{1}^{s}$, which we keep equal to zero. Solving this set of nonlinear equations is delicate. We found that, under normal circumstances, iteration is a stable and quick way to the solution. For a given orientation of the chain, we start with an arbitrary configuration (in fact, for the starting configuration, we use the ground-state configuration of the chain $[19,20]$ ). We then compute the coupling forces $H_{p}^{n}$ and $H_{p}^{s}$, solve the two-by-two equations (33) for $R_{p}^{n}$ and $R_{p}^{s}$, and construct a new set of bond vectors. We repeat the calculation of $H_{p}^{n}$ and $H_{p}^{s}$ for the new configuration and continue the process until the iterative process converges. Iteration leads faster to the ODS than the evolution of the equations, Eq. (32). The results of the two approaches, in any case, coincide.

\section{B. Symmetry breaking in the oriented deterministic state}

The iterative solution of Eq. (33), as well as the decay towards the ODS on the basis of Eq. (32), reveals an interesting phenomenon. To show this, we note that configurations that are invariant under reversal of the chain have vanishing even Rouse modes. It is easy to see that Eqs. (33) preserve this symmetry under iteration. The bond vectors $\mathbf{u}_{n}$ change sign under the operation

$$
n \leftrightarrow N-n
$$

Changing the summation variable from $n$ to $N-n$ in the definition Eq. (14) of the coupling force shows that $\mathbf{H}_{p}$ changes sign for even $p$, but not for odd $p$. This means that if we start the iteration with a configuration that is invariant under reversal, i.e., we start the iteration with only odd $\mathbf{H}_{p}$ on the right hand side of Eq. (33), it leads to a solution that has, once again, only odd Rouse mode components.

The above does not, however, exclude that there are solutions which break the reversal symmetry. The best way to solve for the ODS is to therefore start the iteration with a configuration with a (perturbatively) small even mode, e.g., $\mathbf{R}_{2}$. The perturbation may grow or decrease under successive iterations. We find that for low Weissenberg numbers the perturbation decays to zero, while beyond a critical Weissenberg number $\mathrm{Wi}_{\mathrm{c}}$, the reversal symmetry is broken, i.e., the perturbation grows and saturates at a nonzero value, much as the classic case of a pitchfork bifurcation.

As an example, for a dsDNA chain of length $N=63$ (note: a dsDNA segment of one persistence length corresponds to $N \approx 120$ ), we plot the squared value of the transverse component $R_{2}^{s}$ as a function of the Weissenberg number in Fig. 2. At $\mathrm{Wi}=\mathrm{Wi}_{\mathrm{c}}$ the first nonzero even Rouse modes in the chain appear for $\theta=\pi / 2$ and $\phi=3 \pi / 4$. The coefficient of $R_{2}^{s}$ in Eq. (33),

$$
\zeta_{p}-g s_{x} s_{y}=\zeta_{p}+g(\sin \theta)^{2} \sin \phi \cos \phi,
$$

reaches its smallest value for $\theta=\pi / 2$ and $\phi=3 \pi / 4$, thus leading to the largest value of $R_{2}^{s}$ in the case of symmetry breaking.

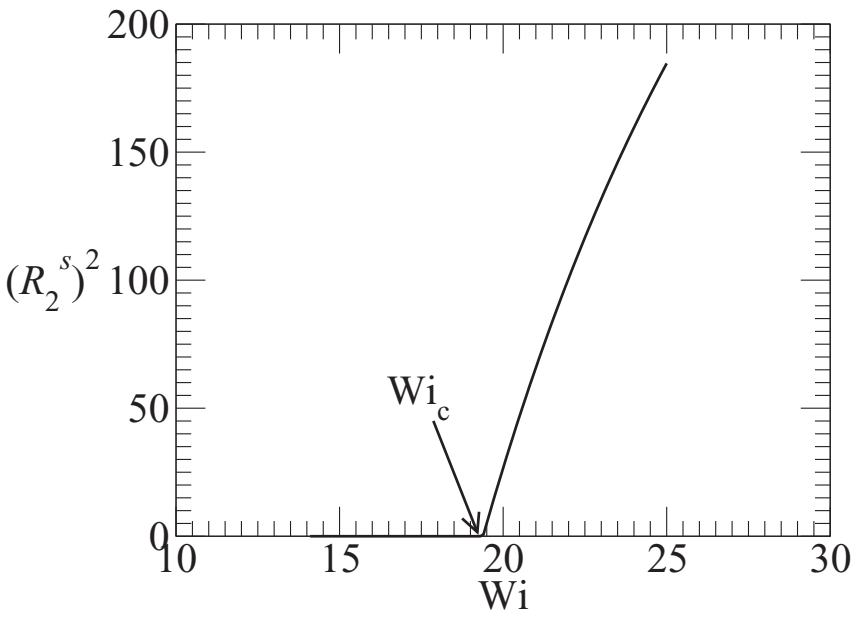

FIG. 2. The squared value of the transverse component $R_{2}^{s}$ as a function of the Weissenberg number for a dsDNA chain of length $N=63$. The critical Weissenberg number is $\mathrm{Wi}_{\mathrm{c}} \approx 19.4$. 

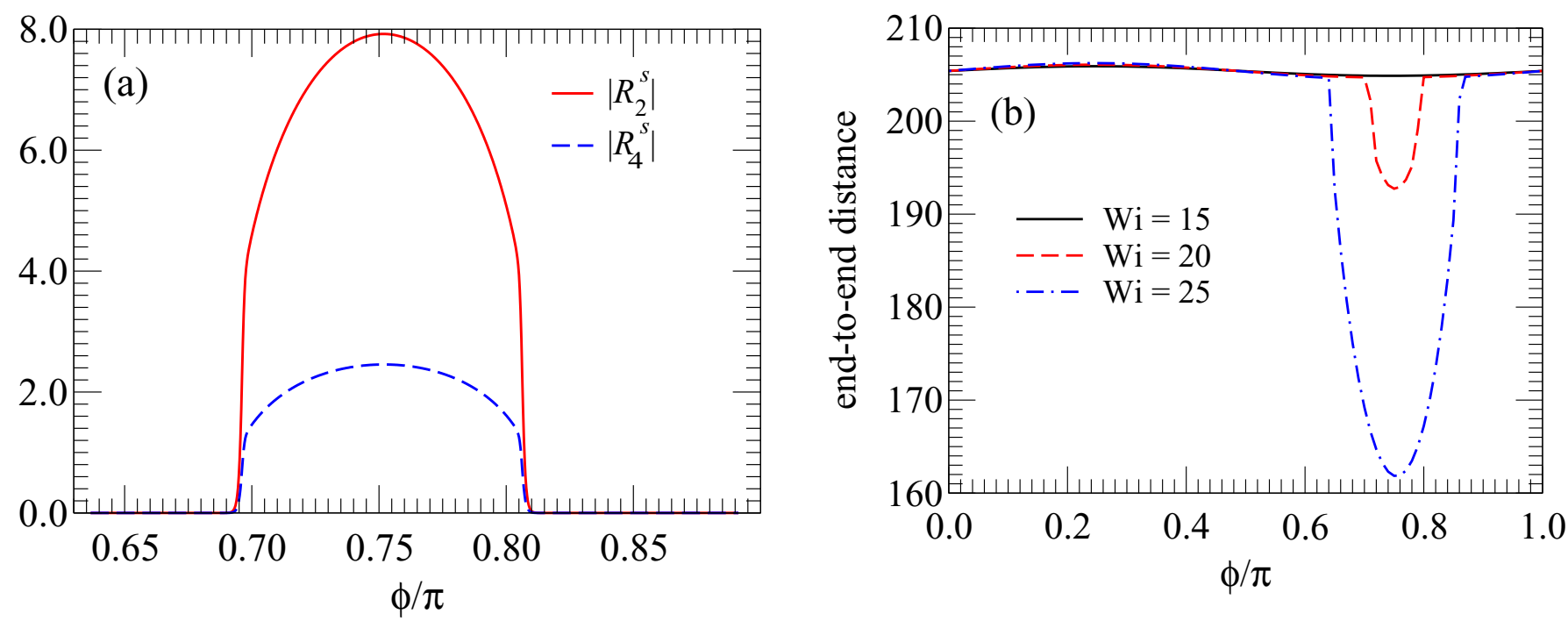

FIG. 3. (a) The appearance of the first two even Rouse modes in a window around the optimal value $\phi=3 \pi / 4$ for $\theta=\pi / 2$ at Wi $=21.3$. (b) The end-to-end distance as a function of $\phi$ for $\theta=\pi / 2$ for some values of $\mathrm{Wi}$ around $\mathrm{Wi}_{\mathrm{c}} \approx 19.4$.

From Fig. 2 we see that the critical Weissenberg number for $\theta=\pi / 2$ and $\phi=3 \pi / 4$ equals $\mathrm{Wi}_{\mathrm{c}} \approx 19.4$ for a dsDNA chain of length $N=63$.

\section{Shapes of the chain and Euler buckling}

The shape of the chain depends on its orientation of the polymer, which comes into the solution through the components of the axes $\hat{\mathbf{n}}$ and $\hat{\mathbf{s}}$. The shear is most effective in the $x-y$ plane, i.e., for $\theta=\pi / 2$. In Fig. 3(a), for $N=63$, we show the value of $\left|R_{2}^{s}\right|$ and $\left|R_{4}^{s}\right|$ as a function of $\phi$ in the neighborhood of the most effective value $\phi=3 \pi / 4$ for $\mathrm{Wi}=21.3$ and $\theta=\pi / 2$ (note: $\mathrm{Wi}_{\mathrm{c}} \approx 19.4$ ). The nonzero value of $\left|R_{p}^{s}\right|$ disappears at $\phi=3 \pi / 4$ when Wi approaches $\mathrm{Wi}_{\mathrm{c}}$ from above.

Further, in order to see the magnitude of the effect, we plot in Fig. 3(b) the behavior of the end-to-end distance of the chain for $N=63$ as a function of $\phi$ for $\theta=\pi / 2$ and for some values of $\mathrm{Wi}$ around the critical Weissenberg number $\mathrm{Wi}_{\mathrm{c}}$. One observes that the end-to-end distance varies only slightly as a function of orientation below $\mathrm{Wi}_{\mathrm{c}}$. Above the $\mathrm{Wi}_{\mathrm{c}}$ a large dip develops around $\phi=3 \pi / 4$, demonstrating that the symmetry breaking goes hand in hand with the so-called Euler buckling of the chain, i.e., the chain folds, which reduces its end-to-end distance.

In order to visually appeal the reader to Euler buckling, we provide a number of snapshots of the chain in the ODS for $N=63$ and $\mathrm{Wi}=100$, confined to the $x-y$ plane in Fig. 4. This large Weissenberg number is well above $\mathrm{Wi}_{\mathrm{c}}$. The region of $\phi$ within $\pi / 2<\phi \leqslant \pi$ is the interesting region, for which we plot the polymer configurations.

To conclude, the ODS configuration of the chain resembles a rigid rod below a critical value $\mathrm{Wi}_{\mathrm{c}}$ of the Weissenberg number. Above this critical value, even though the length of the chain is only about half as that of the persistence length, it breaks the reversal symmetry, much as the classic case of a pitchfork bifurcation. This leads to the development of a region around $\theta=\pi / 2$ and $\phi=3 \pi / 4$, where the chain (Euler) buckles. The buckling gives a large dip in the end-to-end distance.

Finally, we note that the critical $\mathrm{Wi}_{\mathrm{c}}$ depends on the length $N$ of the chain (roughly inversely proportional) and on $v$ (decreasing with $v$ ). Converting it to a critical shear rate $\dot{\gamma}_{c}$ involves also $T^{*}$ [see Eq. (25)].

\section{SHAPES OF A TUMBLING SEMIFLEXIBLE POLYMER: SIMULATIONS}

Our simulations have been performed using an efficient implementation of the semiflexible polymer dynamics [21] of the bead-spring model $[19,20]$.

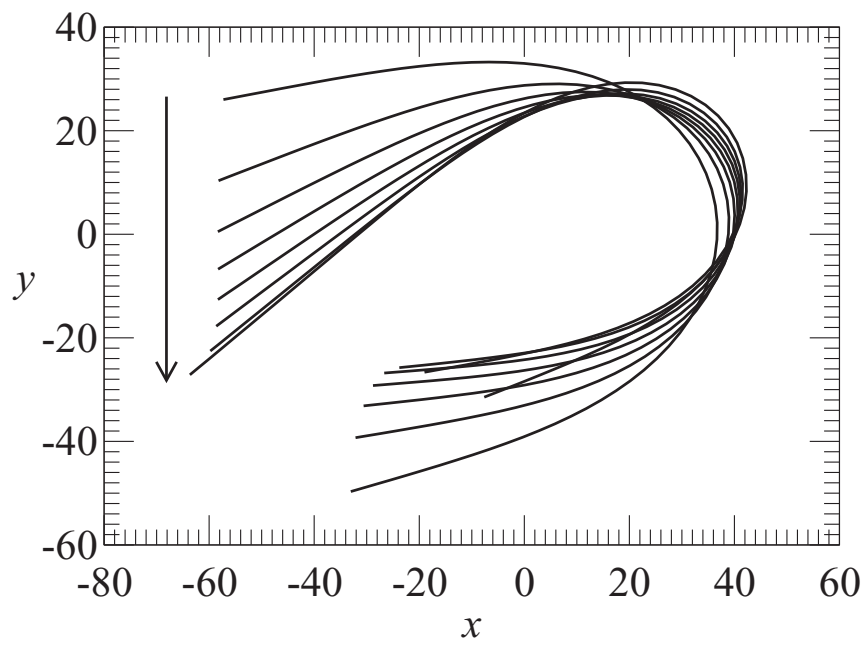

FIG. 4. Shapes of the dsDNA chain of length $N=63$ at $\mathrm{Wi}=$ 100 , demonstrating Euler buckling in the ODS. The shapes are shown on the $x-y$ plane (i.e., $\theta=\pi / 2$ ) for $0.6 \pi \leqslant \phi<\pi$. In the direction of the arrow the shapes correspond to $\phi=0.60 \pi, 0.65 \pi, 0.70 \pi$, $0.75 \pi, 0.80 \pi, 0.85 \pi, 0.90 \pi$, and $0.95 \pi$, respectively. Interestingly, the orientation here defined by the first Rouse mode corresponds closely to the direction of the end-to-end vector. 

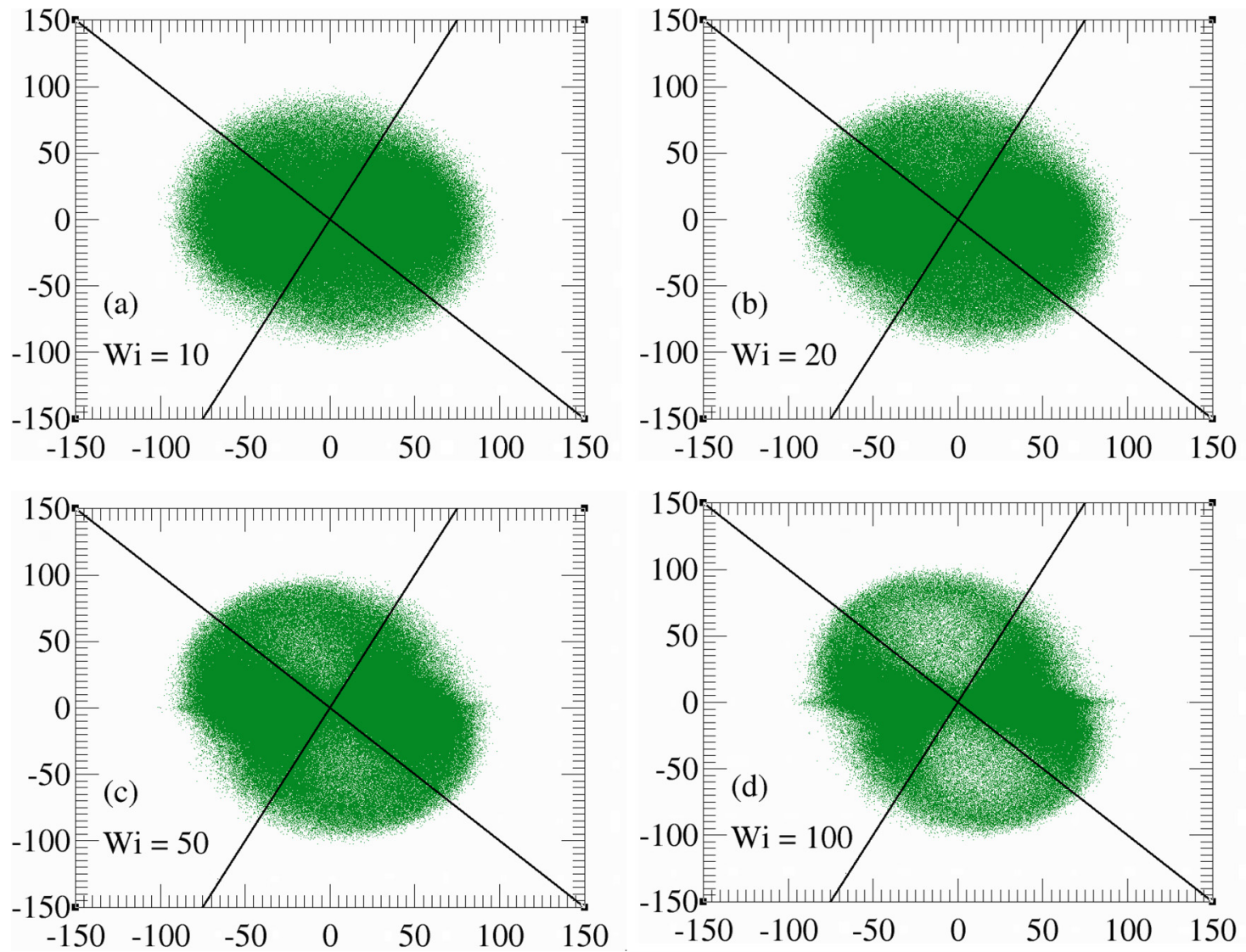

FIG. 5. Scatter plots of $R_{2}^{s}$ as a function of $\phi$ around $\theta=\pi / 2$ at four values of Wi of length $N=63$. In between the solid lines, representing $0.35 \pi<\phi<3 \pi / 4$, we see the probability distribution $P\left(R_{2}^{s}\right)$ of $R_{2}^{s}$ changing with increasing Wi. We take these issues up in Fig. 6 . See also the text for details.

Before we discuss the details of the simulation results, we make the readers aware of the differences between the ODS in Sec. V and the simulations. Thermal noise plays no role in the ODS, while in simulations it does. This implies that although for $\mathrm{Wi}<\mathrm{Wi}_{\mathrm{c}}$ the amplitude of $R_{2}^{s}$ is identically zero in the ODS, we should not expect to find the same in simulations at low Wi values, since in simulations the second Rouse mode will always be kicked up by noise. This calls into question the relevance of the ODS for simulations-in particular, whether the tumbling of the chain is sufficiently slow such that the simulation can explore the neighborhood of the ODS, and thereby follow the characteristics of the ODS. In view of the lack of clarity for an answer to this question, we used the ODS as a guide for the simulations: To be more precise, we focused on the values of $\mathrm{Wi}$ in the range $10 \leqslant \mathrm{Wi} \leqslant 100$ for $N=63$ and sampled the probability distribution of $R_{2}^{s}$ as a function of the orientation.

In simulations for dsDNA of length $N=63$ we recorded 16 million consecutive snapshots of the chain at regular intervals, $\mathbf{R}_{1}$ (for determining the orientation of the chain) and $\mathbf{R}_{2}$, at equal intervals of time, for several values of Wi. The angles $(\theta, \phi)$ for the chain's orientation are determined from the values of $\mathbf{R}_{1}$. We then selected out the snapshots in this slice $|\theta-\pi / 2|=0.1 \mathrm{rad}$, leaving us with 2-3 million snapshots dependent on the value of Wi. In Fig. 5 we show the corresponding scatter plots of $R_{2}^{s}$ as a function of $\phi$ in this slice at four values of $\mathrm{Wi}$. In between the solid lines, representing $0.35 \pi<\phi<3 \pi / 4$, we see two lobes of empty regions developing, signaling that the probability distribution $P\left(R_{2}^{s}\right)$ of $R_{2}^{s}$ for these values of $\phi$ changes with increasing Wi. We note that the values of $\phi$ corresponding to the two solid lines in Fig. 5 are chosen solely by visual inspection, and that the locations of the empty lobes are shifted with respect to the region in $\phi$, for which the ODS exhibits Euler buckling.

In order to further study the change in the probability distribution $P\left(R_{2}^{s}\right)$ of $R_{2}^{s}$, we selected out the data points corresponding to $0.35 \pi<\phi<3 \pi / 4$ in Fig. 5, leaving us 50 000-100 000 data points. From them we constructed the probability distribution $P\left(R_{2}^{s}\right)$. The distributions, corresponding to $\mathrm{Wi}=10,15,20,25,50$, and 100, are shown in Fig. 6. In Fig. 6(a) we see that the unimodal distribution for $\mathrm{Wi}=10$ gradually transforms into a bimodal distribution symmetric around $R_{2}^{s}=0$ for higher $\mathrm{Wi}$. This development is the telltale sign of symmetry breaking, which can also be tracked by the development of the Binder cumulant $B$, defined as

$$
B=1-\frac{\left\langle\left(R_{2}^{s}\right)^{4}\right\rangle}{3\left\langle\left(R_{2}^{s}\right)^{2}\right\rangle},
$$

and shown in Fig. 6(b). The Binder cumulant, originally introduced to study symmetry breaking, attains the value zero when the probability distribution is Gaussian, and reaches the value $2 / 3$ when the symmetry is fully broken, changing the probability distribution into a combination of two symmetric 

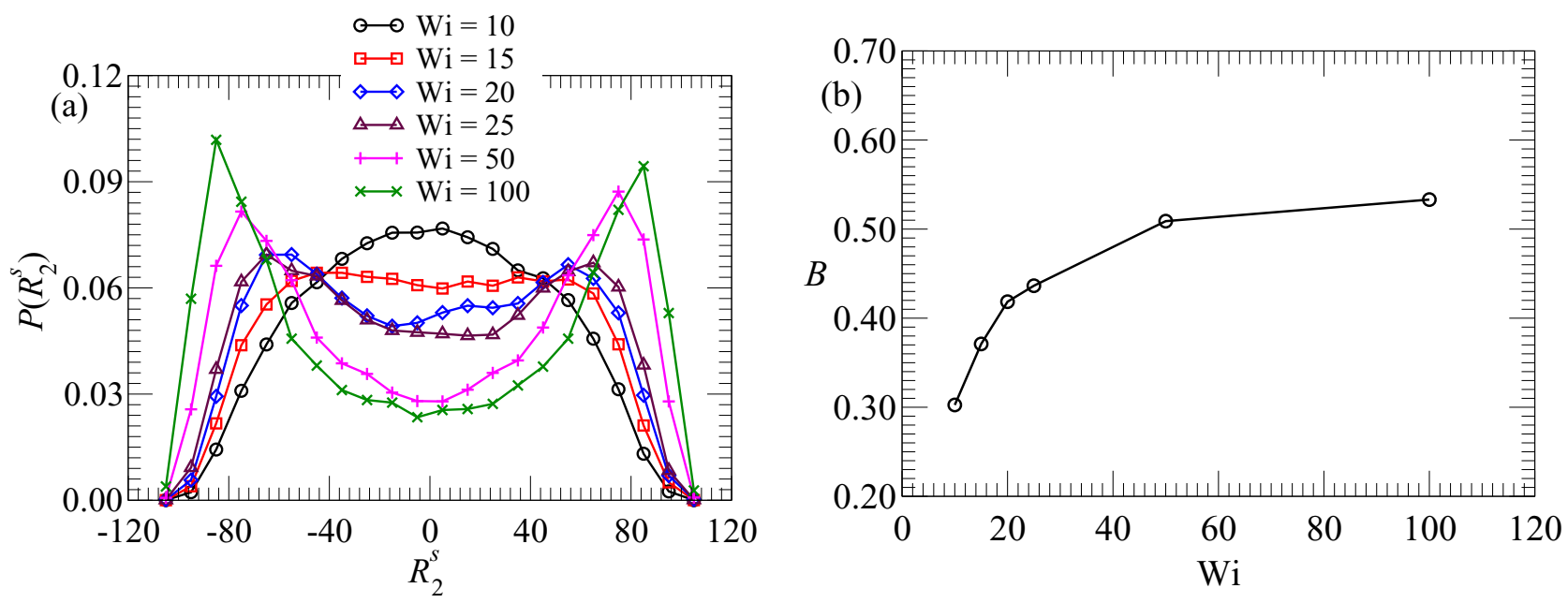

FIG. 6. (a) Probability distribution $P\left(R_{2}^{s}\right)$ of $R_{2}^{s}$ corresponding to $0.35 \pi<\phi<3 \pi / 4$ in Fig. 5 for $N=63$, showing the unimodal distribution for $\mathrm{Wi}=10$ gradually transforming into a bimodal distribution symmetric around $R_{2}^{s}=0$ for higher Wi. (b) The corresponding Binder cumulant $B$, as defined in Eq. (36), which changes from $\approx 0.3$ at $\mathrm{Wi}=10$ to $\approx 0.53$ at $\mathrm{Wi}=100$.

$\delta$ peaks. For the data in Fig. 6(a) we see that the value of $B$ changes from $\approx 0.3$ at $\mathrm{Wi}=10$ to $\approx 0.53$ at $\mathrm{Wi}=100$.

The symmetry breaking is certainly not confined to $N=63$. The same analysis on the simulation data (again, all data points within $|\theta-\pi / 2|=0.1$ and $0.35 \pi<\phi<3 \pi / 4$, with the corresponding figures, analogous to Fig. 6, presented in Fig. 7) reveals symmetry breaking taking place also for $N=31$.

We note that the center of the region where the symmetry breaking takes place is around the values of $\phi \approx 0.55 \pi$, as can be observed from the scatter plots. This is substantially different from the value $\phi=0.75 \pi$, where the onset of buckling takes place in the ODS. The chain tumbles in the direction from $\phi=\pi$ towards $\phi=0$. So the buckling in the simulation lags behind with respect to the ODS. This is likely the result of the slowness by which the buckled state is formed and is broken down. Using Eq. (32) we estimated the time $\Delta \tau$, which is needed to evolve from the ground state (in which the transverse $\mathbf{R}_{2}^{2}=0$ ) to $50 \%$ of its asymptotic value (the ODS), to be of the order $\Delta \tau \simeq 10^{6}$. This translates into a time $\Delta \tilde{\tau} \approx 0.3$ [see Eq. (25)]. In order to put this estimate in perspective, we compare it with the tumbling period $1 / f$ of the rigid rod, which is 1.7 for $\mathrm{Wi}=20$ according to Eq. (29). In other words, the chain indeed travels a sizable fraction of the period in the building-up phase of the buckling, the more so since it rotates faster for the buckling orientations than in the position aligned with the flow.

Thus, to summarize this section, using the theoretical analysis of symmetry breaking as a guide, we have computed the probability distribution $P\left(R_{2}^{s}\right)$ of $R_{2}^{s}$ by simulations of a tumbling dsDNA segment of length $N=63$ and $N=31$. The simulation data have confirmed that symmetry breaking
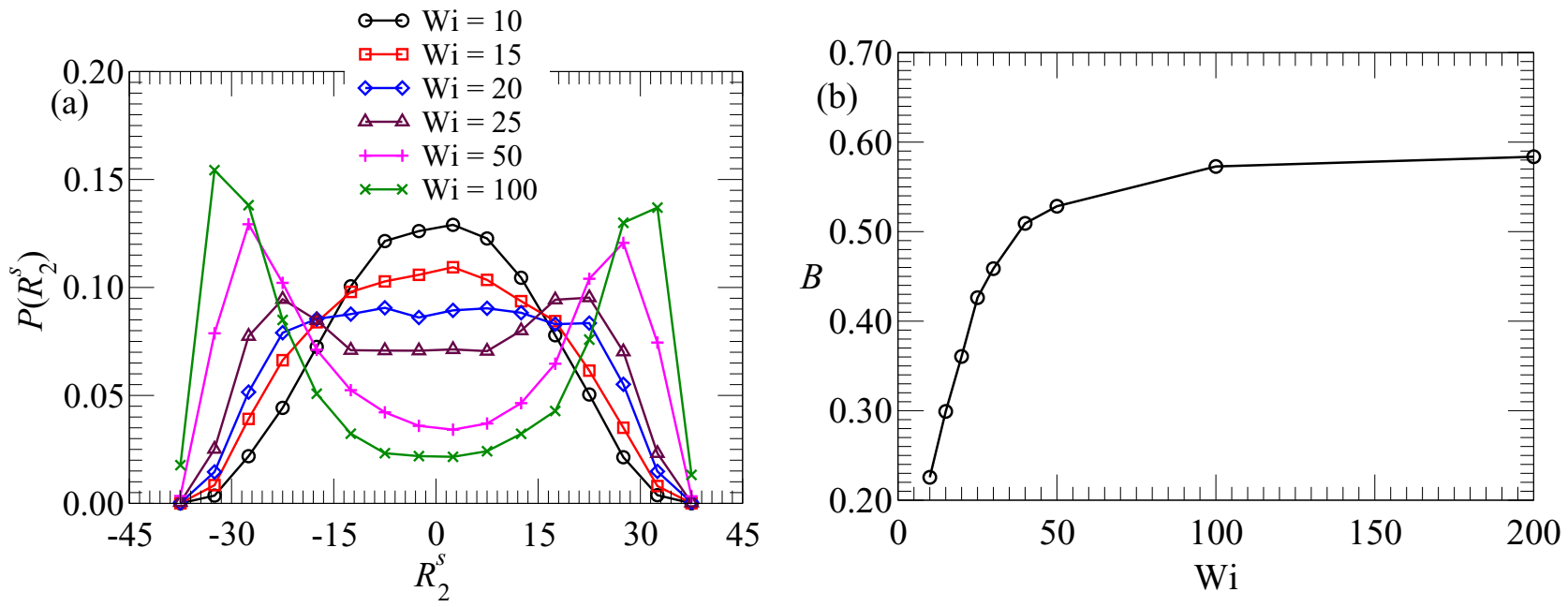

FIG. 7. (a) Probability distribution $P\left(R_{2}^{s}\right)$ of $R_{2}^{s}$ corresponding to $0.35 \pi<\phi<3 \pi / 4$ in Fig. 5 for $N=31$, showing the unimodal distribution for $\mathrm{Wi}=10$ slowly transforming into a bimodal distribution symmetric around $R_{2}^{s}=0$ for higher Wi. (b) The corresponding Binder cumulant $B$, as defined in Eq. (36), which changes from $\approx 0.23$ at $\mathrm{Wi}=10$ to $\approx 0.58$ at $\mathrm{Wi}=200$. 

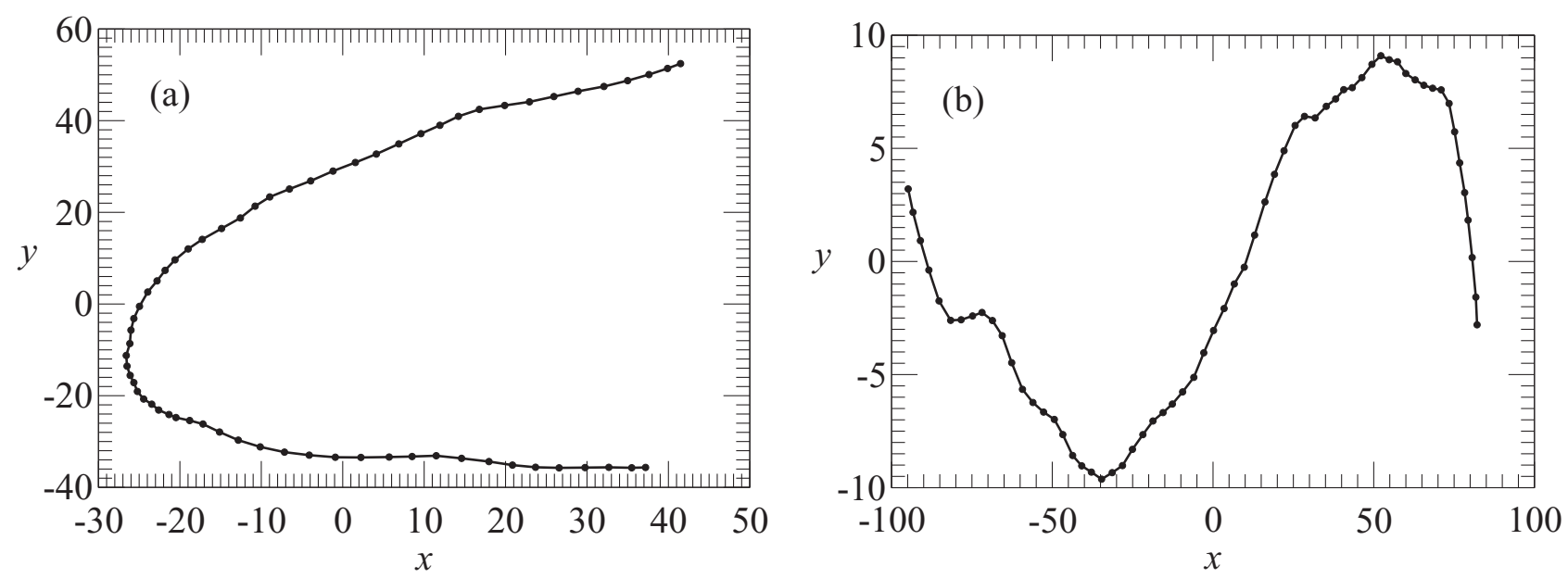

FIG. 8. Simulation snapshots of a tumbling dsDNA chain of length $N=63$ at Wi $=100$, projected on the $x-y$ plane: (a) $U$ shape, (b) $S$ shape.

takes place, showing up as the transition from an unimodal probability distribution $P\left(R_{2}^{s}\right)$ of $R_{2}^{s}$ at $\mathrm{Wi}=10$, transforming into a bimodal distribution symmetric around $R_{2}^{s}=0$, as well as the associated Binder cumulants.

To supplement the above analysis of the simulation data, we show in Fig. 8 two simulation snapshots of a tumbling dsDNA chain of length $N=63$ at $\mathrm{Wi}=100$, projected on the $x-y$ plane, in order to showcase that, akin to the experimental snapshots shown for f-actin in Ref. [16], shear can cause strong deformation even for a chain that is shorter than its persistence length. See the Supplemental Material for a movie of this tumbling chain (that includes both configurations of Fig. 8). In the movie, the center of mass of the chain always remains at the origin of the coordinate system. The movie contains 3000 snapshots, with consecutive snapshots being $\Delta \tau=560$ apart in time. With $\Delta \tau=1$ representing $0.16 \mathrm{ps}$ [21], the full duration of the movie spans $\approx 2.7 \mu \mathrm{s}$ in real time.

\section{CONCLUSION}

Our study focuses on fragments of dsDNA, which are fairly extensible semiflexible polymers. The extensibility of dsDNA implies parameters in our Hamiltonian, which admit mode dynamics with a large time step. The usual workhorse for theoretical studies is the inextensible wormlike chain model for the Hamiltonian, the computer implementation of which is confined to significantly smaller time steps.

Our simulations of a semiflexible polymer (dsDNA fragments smaller than the persistence length) show that their tumbling frequency is given, for the accessible range of Weissenberg numbers $(\mathrm{Wi}<2)$, by the thin rigid-rod formula. Deviations of the tumbling frequency from this formula (Fig. 1) occur at higher Weissenberg numbers. It is theoretically interesting to speculate about the nature of the deviations from the rigid-rod formula, also in view of the observation that the accessible range of Weissenberg numbers is much larger for stiffer and longer polymers, e.g., f-actin. The Weissenberg number for a polymer chain is a product of the shear rate $\dot{\gamma}$ and the rotational diffusion time scale of a rigid rod of the same length as the chain, i.e., L. Consequently, the Weissenberg number $\propto \dot{\gamma} L^{3}$. One persistence length of $\mathrm{f}$-actin is about 200 times longer than one persistence length of dsDNA. In units of persistence length, for the same shear rate, one thus reaches orders of magnitude higher Weissenberg numbers for f-actin than for dsDNA.

In this respect we note that the $\mathrm{Wi}^{2 / 3}$ law for rigid thin rods originates from a singularity that develops in the probability distribution for the orientation in the points $\theta=\pi / 2$ and $\phi=0$ or $\pi$ [18]. The reason is that a thin rigid rod does not feel a torque from the shear in the aligned orientation and only a fluctuation can pull the rod over this stagnation point. A semiflexible polymer, however, always feels a torque due to fluctuations of the other modes (either thermal or buckling), which communicate with the orientation through the coupling force $\mathbf{H}_{1}$. These fluctuations enable Jeffery-like orbits which are characteristic for ellipsoids with a finite aspect ratio in the moments of inertia [24]. The deviations from the thin rigid-rod formula that we see in Fig. 1 do not substantiate the $f \propto \mathrm{Wi}^{3 / 4}$ law reported for inextensible wormlike chains [12].

Using our Hamiltonian we have made a quantitative analysis of the phenomenon of Euler buckling. Fixing the orientation and searching for the configuration which results by turning off the thermal noise yields the oriented deterministic state (ODS). In the ODS we see a sharply defined critical $\mathrm{Wi}_{c}$ above which the buckling occurs. It is a form of symmetry breaking through the occurrence of even modes in the ODS above $\mathrm{Wi}_{c}$.

In the simulations we observe correspondingly a transition in the probability distribution for the even modes, in particular, $R_{2}^{s}$, changing gradually from a unimodal distribution to a bimodal distribution. The simulations show that the formation of the buckled state is a slow process. Therefore, the orientation where the two peaks in the bimodal are most significant lags behind the orientation where the ODS gives the maximum buckling. The buckling is substantiated by characteristic configurations and a movie of the tumbling process. 
[1] C. Bustamante, J. F. Marko, E. D. Siggia, and S. Smith, Science 265, 1599 (1994); J. F. Marko and E. D. Siggia, Macromolecules 28, 8759 (1995).

[2] M. D. Wang, H. Yin, R. Landick, J. Gelles, and S. M. Block, Biophys. J. 72, 1335 (1997).

[3] P. G. de Gennes, J. Chem. Phys. 60, 5030 (1974).

[4] T. T. Perkins, D. E. Smith, R. G. Larson, and S. Chu, Science 268, 83 (1995).

[5] T. T. Perkins, D. E. Smith, and S. Chu, Science 276, 2016 (1997).

[6] D. E. Smith and S. Chu, Science 281, 1335 (1998).

[7] D. E. Smith, H. P. Babcock, and S. Chu, Science 283, 1724 (1999).

[8] P. LeDuc, C. Haber, G. Bao, and D. Wirtz, Nature (London) 399, 564 (1999).

[9] E. Shaqfeh, J. Non-Newtonian Fluid Mech. 130, 1 (2005).

[10] M. Chertkov, I. Kolokolov, V. Lebedev, and K. Turitsyn, J. Fluid Mech. 531, 251 (2005).

[11] A. Celani, A. Puliafito, and K. Turitsyn, Europhys. Lett. 70, 464 (2005).

[12] P. S. Lang, B. Obermayer, and E. Frey, Phys. Rev. E 89, 022606 (2014).
[13] P. S. Doyle, B. Ladoux, and J.-L. Viovy, Phys. Rev. Lett. 84, 4769 (2000).

[14] R. E. Teixeira, H. P. Babcock, E. S. Shaqfeh, and S. Chu, Macromolecules 38, 581 (2005).

[15] C. M. Schroeder, R. E. Teixeira, E. S. G. Shaqfeh, and S. Chu, Phys. Rev. Lett. 95, 018301 (2005).

[16] M. Harasim, B. Wunderlich, O. Peleg, M. Kröger, and A. R. Bausch, Phys. Rev. Lett. 110, 108302 (2013).

[17] G. Jeffery, Proc. R. Soc. London, Ser. A 102, 161 (1922).

[18] J. M. J. van Leeuwen and H. W. J. Blöte, J. Stat. Mech. (2014) P09007.

[19] G. T. Barkema and J. M. J. van Leeuwen, J. Stat. Mech. (2012) P12019.

[20] G. T. Barkema, D. Panja, and J. M. J. van Leeuwen, J. Stat. Mech. (2014) P11008.

[21] D. Panja, G. T. Barkema, and J. M. J. van Leeuwen, Phys. Rev. E 92, 032603 (2015).

[22] P. E. Rouse, J. Chem. Phys. 21, 1272 (1953).

[23] See Supplemental Material at http://link.aps.org/supplemental/ 10.1103/PhysRevE.93.042501 for a movie of a tumbling dsDNA chain (that includes both configurations of Fig. 8).

[24] J. M. Burgers, Proc., K. Ned. Akad. Wet. XVI, 113 (1938). 